\title{
छூ \\ Influence of space-charge fields on the cooling process of muon beams
}

\author{
Diktys Stratakis and Robert B. Palmer \\ Brookhaven National Laboratory, Upton, New York 11973, USA \\ David P. Grote \\ Lawrence Livermore National Laboratory, Livermore, California 94550, USA
}

(Received 15 November 2014; published 7 April 2015)

\begin{abstract}
Obtaining muon beams with high density in 6D phase space is essential for realization of muon colliders, neutrino factories based on accelerated muons beams and other experiments involving muons. While several schemes to compress the beam phase space by means of muon cooling have been proposed, very little is known about the impact of particle-particle interactions in the whole design. In this paper, we examine the influence of space-charge fields on the cooling process of muon beams. We show that the cooling efficiency decreases with the degree of intensity, leading to emittance growth and particle loss for beams with large intensities. We further show that the emittance growth is only longitudinal and present a space-charge compensation solution by means of increasing the rf gradient. With the aid of numerical simulations, we obtain a quantitative relationship between the required compensation gradient and bunch charge and compare our results to earlier theoretical findings.
\end{abstract}

DOI: 10.1103/PhysRevSTAB.18.044201

PACS numbers: 29.20.Ej, 41.75.Lx

\section{INTRODUCTION}

Muons are "heavy electrons" that have negligible synchrotron radiation and beamsstrahlung emission, and can be accelerated and stored in multiturn recirculating devices or rings [1,2]. Recently, interest has increased in the possibility of using muons in high-energy physics as the colliding particles in $\mu^{+}-\mu^{-}$colliders [3]. The liabilities of muons are that they decay fast and that they are created through decay into a diffuse phase space. As a result, the volume of the six-dimensional (6D) phase space of a muon beam must be rapidly reduced several orders of magnitude in order to be able to further accelerate it.

Ionization cooling [4-6] is currently the only feasible option within the very short muon lifetime $\left(\tau_{0}=2.19 \mu \mathrm{s}\right)$ and a complete scheme for cooling a muon beam sufficiently for use in a muon collider has been previously described [7]. This scheme uses separate 6D ionization cooling channels for the two signs of the particle charge. In each, a channel first reduces the emittance of a train of muon bunches until they can be injected into a bunchmerging system [8]. The single muon bunches, one of each sign, are then sent through a second 6D cooling channel where the transverse emittance is reduced as much as possible and the longitudinal emittance is cooled to a value below that needed for the collider. If necessary, the beam can then be recombined and sent through a final cooling

Published by the American Physical Society under the terms of the Creative Commons Attribution 3.0 License. Further distribution of this work must maintain attribution to the author $(s)$ and the published article's title, journal citation, and DOI. channel [9] using high-field solenoids that cools the transverse emittance to the required values for the collider while allowing the longitudinal emittance to grow. Figure 1(a) shows the longitudinal versus the transverse emittances as they evolve from their initial high values to the final desired values for a muon collider [10].

Ionization cooling is achieved by reducing the beam momentum through ionization energy loss in absorbers and replenishing the momentum loss only in the longitudinal direction through $\mathrm{rf}$ cavities. This mechanism can effectively reduce the transverse phase space of a beam in the same way as radiation damping does to an electron beam. However it does not effectively cool the longitudinal momentum spread because the energy-loss rate is not sensitive to beam momentum except for very low-energy muons. In order to reduce the longitudinal emittance, the so-called "emittance exchange" [11] technique has been proposed to be used and is commonly introduced in the design work. With this technique, a dispersive beam is passed through a discrete $[12,13]$ or continuous absorber [14] in such a way that the high-energy particles traverse more material than the low-energy particles. The net result is a reduction of the longitudinal emittance at the cost of simultaneously increasing the transverse emittance. By controlling the amount of emittance exchange the sixdimensional emittance can be reduced.

While there has been significant progress in the design and simulation of ionization cooling channels in the past decade, very little is known about the impact of particleparticle interactions in the whole design. Numerical simulations [15] predict that at the end of the post-merge 6D cooling channel, the muon bunch has $5 \times 10^{12}$ muons, an 


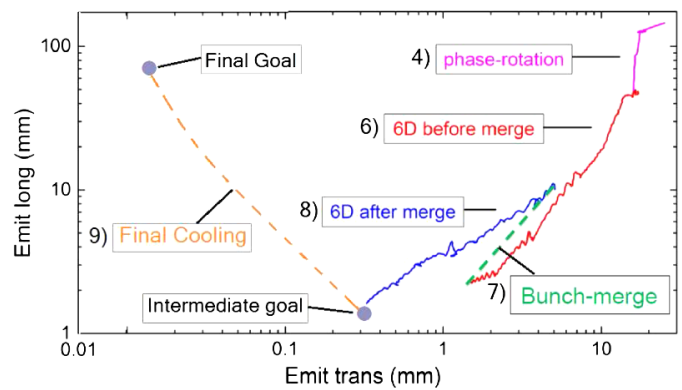

(a)

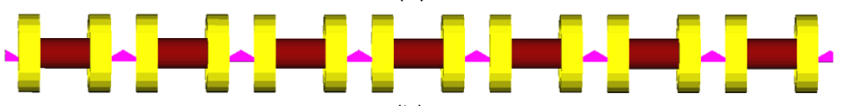

(b)

FIG. 1. (a) Simulated evolution of emittances from start of phase rotation to start of final cooling, and projected evolution through the final cooling (dashed). Note that this study focuses on the post-merge $6 \mathrm{D}$ cooling channel only. For a muon collider, the desired transverse and longitudinal normalized emittances at the end of this channel are $\leq 0.30$ and $\leq 1.0 \mathrm{~mm}$, respectively. (b) Conceptual design of our 6D ionization cooling channel. Note that the large yellow cylinders are solenoids, the small red cylinders are the active volume of the rf cavities, and the magenta wedges are absorbers.

average momentum of $200 \mathrm{MeV} / c$ and rms length of $2 \mathrm{~cm}$. With this beam current, and relativity low momentum, it is likely that the cooling performance of the channel will be limited by space charge, and it is uncertain whether we can achieve the micron-scale emittances required for a muon collider. In particular, a theoretical study by Palmer et al. [16] has indicated that space-charge forces are especially dominant for short bunches resulting from longitudinal cooling to normalized emittances $\leq 2 \mathrm{~mm}$. Quantitatively, the ratio between the Laslett tune shift [17] and betatron tune, $\Delta \nu_{S C} / \nu$ was estimated [18] to be near 0.11 at the end of the channel, which is comparable to the $\sim 0.15$ for the space-charge dominated Proton Synchrotron Booster [19] of the Large Hadron Collider. The three interesting questions then are: what is the minimum emittance that can be achieved, how it scales with the bunch charge and whether a space-charge compensation solution, by means of increasing the rf gradient, is applicable.

In this paper, we numerically examine the influence of space-charge fields on the cooling process of muon beams with Warp [20], a well-established code for space-charge simulation that is properly modified so that it fully incorporates all basic particle-matter interaction physical processes such as energy loss, scattering, straggling and muon decay. We show that the cooling efficiency decreases with the degree of intensity, leading to emittance growth and particle loss for beams with large intensities. We further show that the deterioration of the performance is primarily caused by the longitudinal space-charge force and that the rf gradient can play a key role for space-charge compensation in ionization cooling channels. With the aid of numerical simulations we establish a quantitative relationship between the required compensation gradient and bunch charge. We find that for a muon collider, in order to obtain a longitudinal emittance that is $<1.0 \mathrm{~mm}$, the rf gradient of a $805 \mathrm{MHz}$ cavity needs to surpass the challenging limit of $32.5 \mathrm{MV} / \mathrm{m}$.

The reminder of the paper is structured as follows: In Sec. II, we give an overview of the ionization cooling concept. In Sec. III, we provide design details of the ionization cooling channel. In Sec. IV we numerically evaluate the influence of space-charge fields on the cooling process of the aforementioned channel for a wide range of beam intensities. We also discuss a potential space-charge compensation solution. Finally, we present our conclusions in Sec. V. We note that this paper focuses on the $6 \mathrm{D}$ cooling channel after the merge only.

\section{IONIZATION COOLING THEORY}

The initial muon $6 \mathrm{D}$ emittance is $\sim 10^{6}$ times the specified final goal, so emittance reduction (cooling) is necessary. In ionization cooling, particles pass through a material medium and lose energy through ionization interactions, and this is followed by beam reacceleration in rf cavities. The losses are parallel to the particle motion, and therefore include transverse and longitudinal momentum losses; the reacceleration restores only longitudinal momentum. The net loss of transverse momentum reduces particle emittances and thus cools the beam. However, the random process of multiple scattering in the material medium increases the rms beam divergence, adding a heating term which must be controlled in a complete cooling system. When the competing process of energy loss and multiple scattering become equal, the beam reaches an equilibrium emittance given by [21]

$$
\varepsilon_{N, \mathrm{eq}}=\frac{\beta_{T} E_{s}^{2}}{2 \beta m_{\mu} c^{2} L_{R}\left|\frac{d E_{\mu}}{d s}\right|},
$$

where $\varepsilon_{N \text {,eq }}$ is the normalized transverse emittance, $E_{\mu}$ is the muon energy, $m_{\mu}$ is the muon mass, $\beta_{T}$ is the transverse betatron function within a discrete absorber, $\beta$ is the particle velocity, $c$ is the speed of light, $d E_{\mu} / d s$ is the energy loss per unit length, $L_{R}$ is the radiation length of the material and $E_{s}$ is the characteristic scattering energy $(\sim 13.6 \mathrm{MeV})$. One wants to use absorbers where the product of radiation length and the energy loss rate is large. Liquid hydrogen $(\mathrm{LH})$ and lithium hydride $(\mathrm{LiH})$ are the best choices. The only other parameter that is under our control is $\beta_{T}$, which we want to keep as small as possible over the length of the absorber. While ionization cooling can be accomplished at any energy, if done at higher momenta, excessive acceleration is needed. On the other hand, at momenta below $200 \mathrm{MeV} / c$, the rising energy loss gives excessive 
longitudinal emittance growth. Therefore, a momentum near $200 \mathrm{MeV} / c$ is optimal.

Simple energy loss cools transversely, but not longitudinally (energy-time space). For longitudinal cooling we must use geometries yielding greater energy loss at higher momenta than lower. This can be done by placing a wedge absorber in a region with dispersion so that higher momentum particles see more energy loss than the lower ones. The same can be achieved in a channel with a continuous absorbing material by having longer path lengths for higher momentum particles, thus lowering their energy more. The present study considers lattices with wedge absorbers only.

The cooling performance of a channel is conveniently evaluated using the cooling efficiency factor, $Q_{6 D}$ [22], which is defined as the rate of change of $6 \mathrm{D}$ emittance divided by the rate of change of the number of particles in the beam. In a given lattice $Q_{6 D}$ starts of small due to losses from initial matching, then rises to a large value $\left(Q_{6 D}=10\right.$ is typical for the channels discussed here) and finally falls as the emittance of the beam approaches its equilibrium value. We found [23] that good cooling efficiency requires that the channel is tapered. In that case when $Q_{6 D}$ starts to fall off, the lattice is modified to reduce the beta function. This ensures that the beam emittance is always large compared with the equilibrium emittance.

\section{COOLING LATTICE DESIGN}

Figure 1(b) shows a sketch of a potential 6D ionization cooling channel for a muon collider. The channel consists of a series of identical cells with two or four solenoids in each cell with opposite polarity to provide transverse focusing. The coils (yellow) have alternating tilts to generate dipole fields that give the needed dispersions at the wedge absorbers. The coils are not evenly spaced; those on either side of the wedge absorber (magenta) are closer together in order to increase the focusing at the absorber and thus minimizing the beta function at that location. The relative amount of cooling can be adjusted by changing the opening angle and transverse location of the wedge. A series of rf cavities (dark red) are used to restore the momentum along the longitudinal axis.

As noted earlier, good cooling efficiency requires the channel to be tapered. As a result, parameters such as the cell length, focusing strength and rf frequency change from stage to stage based on the emittance reduction rate and transmission with the purpose to maintain a beam emittance that is always larger from the equilibrium emittance. We consider a 17-stage channel, where each stage consists of a sequence of identical cells and some of the main lattice parameters are summarized in Table I [24]. At the first stage of the channel, the focusing will be relatively weak to avoid excessive angular divergence that can arise from the large transverse emittance of the initial beam. This stage is then terminated and we couple into the next stage that has a lower beta. This is achieved by simultaneously scaling down the cell dimensions and raising the strength of the on-axis solenoidal field.

Figure 2(a) shows the side view of one cell of the first stage of the channel (stage 1). This stage consists of a sequence of eight identical $275 \mathrm{~cm}$ cells, each containing four $47.0 \mathrm{~cm}$-long $201 \mathrm{MHz}$ pillbox cavities, and one wedge-shaped LH absorber to assure energy loss. Moreover, each cell contains two solenoid coils of opposite polarity, yielding an approximate sinusoidal variation of the

TABLE I. Main lattice parameters of the post-merge 6D cooling lattice of a muon collider. The rf gradient is the peak gradient within the cavity.

\begin{tabular}{lccccccccccc}
\hline \hline $\begin{array}{l}\text { Stage } \\
\text { No. }\end{array}$ & $\begin{array}{c}\text { Cell length } \\
{[\mathrm{cm}]}\end{array}$ & $\begin{array}{c}\text { No. } \\
\text { cells }\end{array}$ & $\begin{aligned} \text { rf frequency } \\
{[\mathrm{MHz}] }\end{aligned}$ & $\begin{array}{c}\text { rf gradient } \\
{[\mathrm{MV} / \mathrm{m}]}\end{array}$ & rf No. & rf length $[\mathrm{cm}]$ & $\boldsymbol{B}_{0}(\mathrm{~T})$ & $\boldsymbol{\beta}_{\boldsymbol{T}, 0}(\mathrm{~cm})$ & $\begin{array}{c}\text { Absorber } \\
\text { material }\end{array}$ & $\begin{array}{c}\text { Absorber } \\
\text { length }[\mathrm{cm}]\end{array}$ & $\delta$ \\
\hline 1 & 275.00 & 8 & 201.0 & 15.5 & 4 & 47.00 & 2.8 & 39 & LH & 42.60 & 1.0300 \\
2 & 235.70 & 8 & 235.0 & 15.5 & 4 & 40.00 & 3.1 & 35 & LH & 36.52 & 1.0260 \\
3 & 202.10 & 9 & 274.0 & 15.5 & 4 & 34.50 & 3.6 & 29 & LH & 31.31 & 1.0220 \\
4 & 173.20 & 11 & 319.0 & 15.5 & 4 & 29.60 & 4.2 & 25 & LH & 26.84 & 1.0190 \\
5 & 148.50 & 12 & 372.0 & 15.5 & 4 & 25.40 & 4.9 & 21 & LH & 23.00 & 1.0160 \\
6 & 127.30 & 15 & 402.0 & 15.5 & 4 & 21.75 & 5.7 & 18 & LH & 19.72 & 1.0130 \\
7 & 109.10 & 17 & 507.0 & 15.5 & 4 & 18.65 & 6.7 & 16 & LH & 16.90 & 1.0103 \\
8 & 93.55 & 20 & 591.0 & 15.5 & 4 & 16.00 & 7.8 & 13 & LH & 14.49 & 1.0088 \\
9 & 80.20 & 24 & 690.0 & 15.5 & 4 & 13.70 & 9.1 & 11 & LH & 12.42 & 1.0075 \\
10 & 68.75 & 40 & 805.0 & 15.5 & 4 & 11.75 & 10.6 & 10 & LH & 10.65 & 1.0065 \\
11 & 68.75 & 40 & 805.0 & 15.5 & 4 & 11.75 & 10.9 & 8.2 & LH & 10.65 & 1.0065 \\
12 & 68.75 & 40 & 805.0 & 15.5 & 4 & 11.75 & 11.8 & 6.9 & LH & 10.65 & 1.0065 \\
13 & 68.75 & 40 & 805.0 & 15.5 & 4 & 11.75 & 12.3 & 5.9 & LH & 10.65 & 1.0065 \\
14 & 68.75 & 40 & 805.0 & 15.5 & 4 & 11.75 & 13.1 & 4.9 & LH & 10.65 & 1.0065 \\
15 & 68.75 & 40 & 805.0 & 15.5 & 4 & 11.75 & 13.9 & 4.1 & LiH & 1.92 & 1.0060 \\
16 & 68.75 & 51 & 805.0 & 15.5 & 4 & 11.75 & 14.9 & 3.4 & LiH & 1.92 & 1.0060 \\
17 & 68.75 & 67 & 805.0 & 15.5 & 4 & 11.75 & 15.8 & 2.8 & LiH & 1.92 & 1.0040 \\
\hline \hline
\end{tabular}




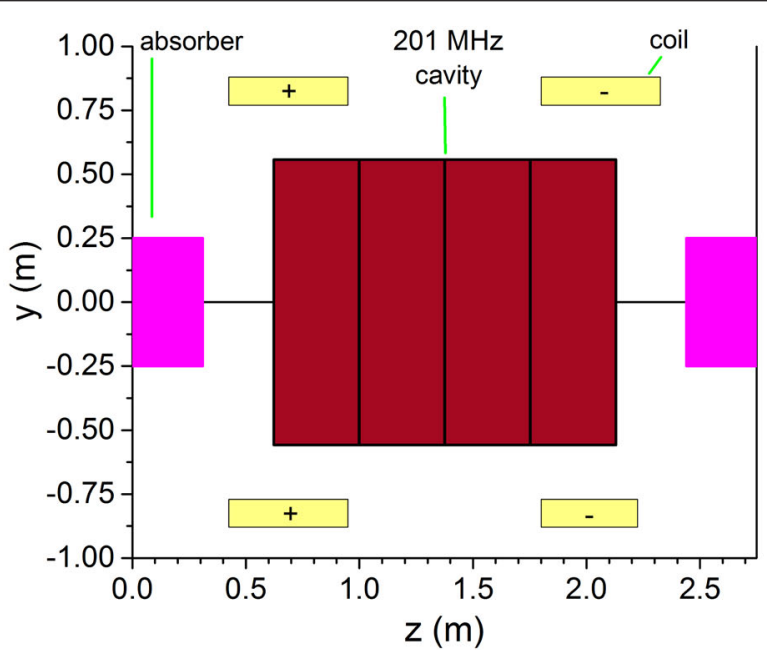

(a)

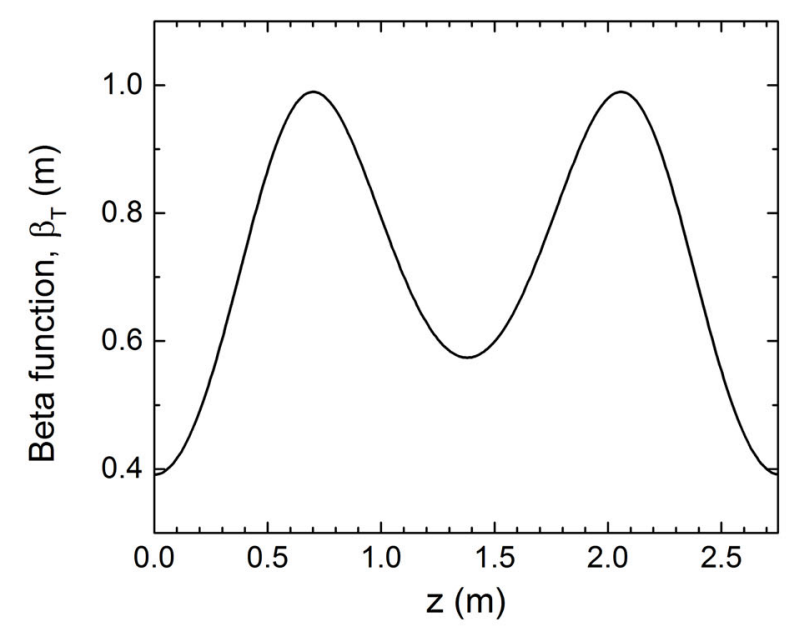

(c)

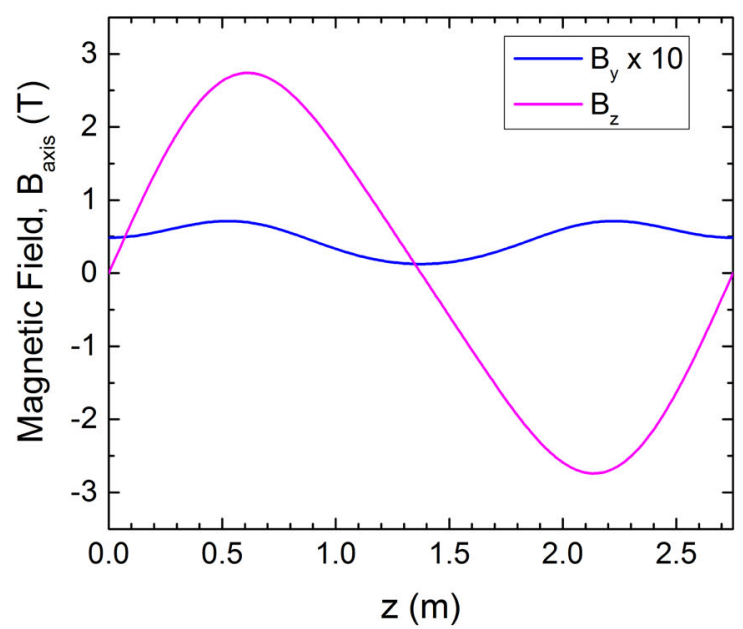

(b)

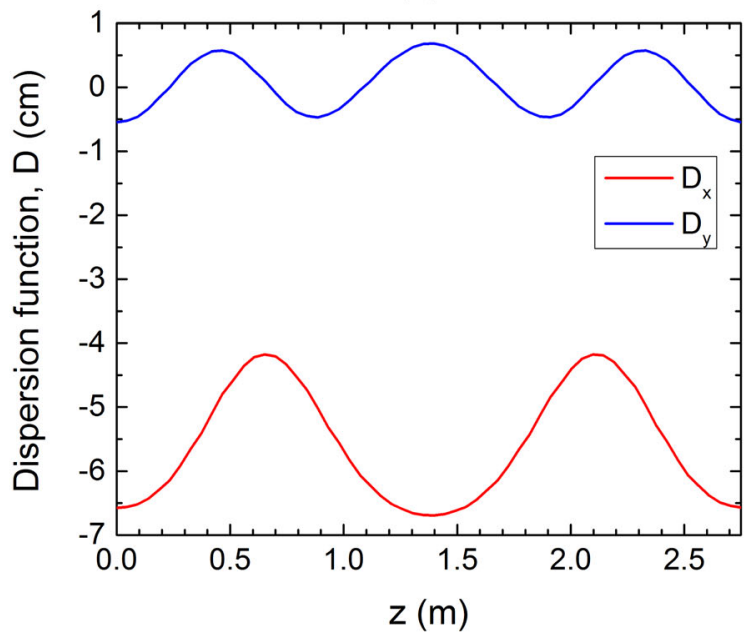

(d)

FIG. 2. Lattice characteristics of the simulated 6D cooling channel: (a) Side view of one cell for the first stage (stage 1). Note that the tilt of the coils along the $y z$ plane is not shown; (b) magnetic field versus $z$. The vertical field $B_{y}$ is shown in blue, and the longitudinal field $B_{z}$ is shown in magenta. The horizontal axial field $B_{x}$ is zero; (c) beta function versus $z$ at the reference momentum of $200 \mathrm{MeV} / c$; (d) dispersion versus $z$ at $200 \mathrm{MeV} / c$.

axial magnetic field in the channel which is depicted in Fig. 2(b). The tilt of the solenoids along the $y z$ plane is adjusted to provide a peak transverse field of $0.07 \mathrm{~T}$ on-axis in order to provide adequate emittance exchange. One can see from Fig. 2(c) that the minimum value of the transverse betatron function $\beta_{T, 0}$, is $39.0 \mathrm{~cm}$, at $200 \mathrm{MeV} / c$, and occurs at the absorber center. In principle, placing the absorber on that location would enhance the cooling rate compared to any other location in the cell, simply because the equilibrium emittance is proportional to the beta function [see Eq. (1)]. Finally, Fig. 2(d) displays the dispersion function in $x$ (red) and $y$ (blue) versus position, $z$. In a similar fashion to Ref. [25], the dispersion is dominant in one axis, which is the $x$ direction for our case.

Figure 3 shows the last cooling stage (stage 17) that is designed for late stage of cooling with the goal to produce a beam with a normalized transverse rms emittance $\leq 0.30 \mathrm{~mm}$ and a normalized longitudinal rms emittance $\leq 1.0 \mathrm{~mm}$ as required for a muon collider [7]. In order to reach this goal, the transverse beta function at the absorber has to be reduced from $\sim 39.0$ to $\sim 2.8 \mathrm{~cm}$. This reduction, without changing the essential dynamics, is achieved by scaling down the cell length (from 275.0 to $68.75 \mathrm{~cm}$ ) and raising the peak axial field (from 2.8 to $15.8 \mathrm{~T}$ ). Stage 17 consists of 67 cells, each containing four $805 \mathrm{MHz}$ cavities and one $\mathrm{LiH}$ wedge absorber to produce the energy loss as before. The solid blue curve depicts the variation of the axial magnetic field along the cell. By carefully examining Figs. 2 and 3 the following points are noteworthy: First, the peak axial magnetic field for stage 17 is 5.6 times larger than in stage 1 . As a result, the beta function at the absorber shrinks to $2.8 \mathrm{~cm}$ and the equilibrium emittance becomes $\varepsilon_{N \text {,eq }} \approx 0.18 \mathrm{~mm}$ which is adequate enough to reduce the normalized rms emittance to the desired values, i.e., 


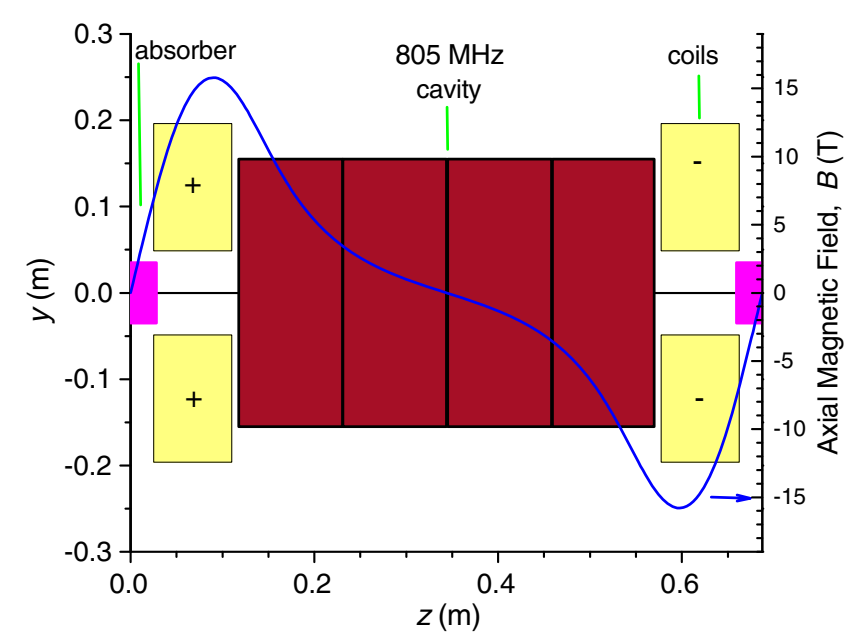

FIG. 3. Side view of one cell of the last stage (stage 17). The solid blue curve shows the axial magnetic field at various positions along the cell. Note that the tilt of the coils along the $y z$ plane is not shown.

$<0.30 \mathrm{~mm}$. Moreover, the frequency of the last cooling stage is 4 times higher compared to stage 1 . The increase in frequency reduces the relative fraction of length needed for the $\mathrm{rf}$ and gives more space for solenoidal coils. Most importantly, it allows the coils to be placed closer to the beam axis, consequently reducing the beta function and thus improving the cooling performance.

Table I exhibits the transverse beta function at the absorber center and peak-on-axis magnetic field for all stages. The beta function varies from 39.0 to $2.8 \mathrm{~cm}$ while the peak on-axis magnetic field, $B_{0}$ increases progressively from 2.8 to $15.8 \mathrm{~T}$. The field becomes progressively stronger in order to enhance the cooling rate by suppressing the beta function at the absorber and hence the equilibrium emittance. An unfortunate implication of the smaller beta function is its faster rise when moving away from the absorber. This makes the use of LH impractical near the end of the channel and we switched to $\mathrm{LiH}$ for the last three cooling stages. Note that in Table I, "rf length" is the length of each cavity and that all stages contain four cavities per cell. Note further that the rf gradient does not scale with frequency but rather remains fixed at $15.5 \mathrm{MV} / \mathrm{m}$ to avoid possible limitations due to breakdown in a magnetic field [26]. The corresponding accelerating phase is $34 \mathrm{deg}$. as this value provides the highest possible $Q_{6 D}$ for each stage.

\section{INFLUENCE OF SPACE-CHARGE FIELDS}

ICOOL [27] is routinely used by the muon acceleration program [28] to study the performance of ionization cooling lattices. The code fully incorporates all basic particlematter-interaction physical processes such as energy loss, straggling, and multiple scattering. However, it excludes interactions between individual particles. Note that as the beam propagates through the channel, its size shrinks by at least 1 order of magnitude in both transverse and longitudinal directions. As the size of the beam decreases, the density of particles increases and it is likely that Coulomb repulsion (space-charge effect) in the beam becomes significant during the last stages of the cooling channel.

In order to address the above issue, we numerically examine the influence of space-charge fields on the cooling process using the particle in-cell code Warp. Warp was originally developed to simulate space-charge-dominated beam dynamics in induction accelerators for heavy-ion fusion (HIF). The code now has a broad user base and is being applied to projects both within [20] and far removed from the HIF community [29]. In our approach, a layer was added to Warp to read in and parse ICOOL input files allowing identical problem setup. Note though that Warp operates with time as the independent variable whereas ICOOL uses space, so there are small numerical differences in the calculations. During the calculation, Warp handles all operations except for the interaction of the muons with the material in the absorber. The material interaction is handled by calls to the appropriate ICOOL routines during the calculation. For each stage, we generated 2D cylindrical field maps by superimposing the fields from all solenoids in the cell and its neighbor cells. The rf cavities were modeled using cylindrical pillboxes running in the TM010 mode and a reference particle was used to determine each cavity's relative phase. The aperture was gradually decreasing along the channel in order to accommodate the higher frequency rf cavities for the later stages. The absorber material was LH in the early stages, enclosed in thin aluminum windows, and $\mathrm{LiH}$ in the late ones.

A simplified cooling model of the channel was used for the initial simulation with Warp. Specifically, ionization cooling was simulated in a series of straight lattices with block absorbers and no tilt. Hence, there was no bending field dispersion, and this arrangement produced transverse cooling, only. Emittance exchange was simulated via linear matrix acting on $\left(\begin{array}{llllll}x & x^{\prime} & y & y^{\prime} & \sigma_{z} & \sigma_{p} / p\end{array}\right)$ introduced before each cell. The matrix used was of the form

$$
M=\left(\begin{array}{cccccc}
1 & 0 & 0 & 0 & 0 & 0 \\
0 & 1+\delta & 0 & 0 & 0 & 0 \\
0 & 0 & 1 & 0 & 0 & 0 \\
0 & 0 & 0 & 1+\delta & 0 & 0 \\
0 & 0 & 0 & 0 & 1 & 0 \\
0 & 0 & 0 & 0 & 0 & 1-2 \delta
\end{array}\right)
$$

The amount of exchange was controlled using a parameter $\delta$ (see Table I), which reduced the momentum spread, $\sigma_{p}$, and simultaneously increased the angular divergence $\left(x^{\prime}, y^{\prime}\right)$ of the beam. The value of the control parameter, $\delta$, was chosen with the objective to provide the maximum cooling efficiency factor for each stage. This 2D 


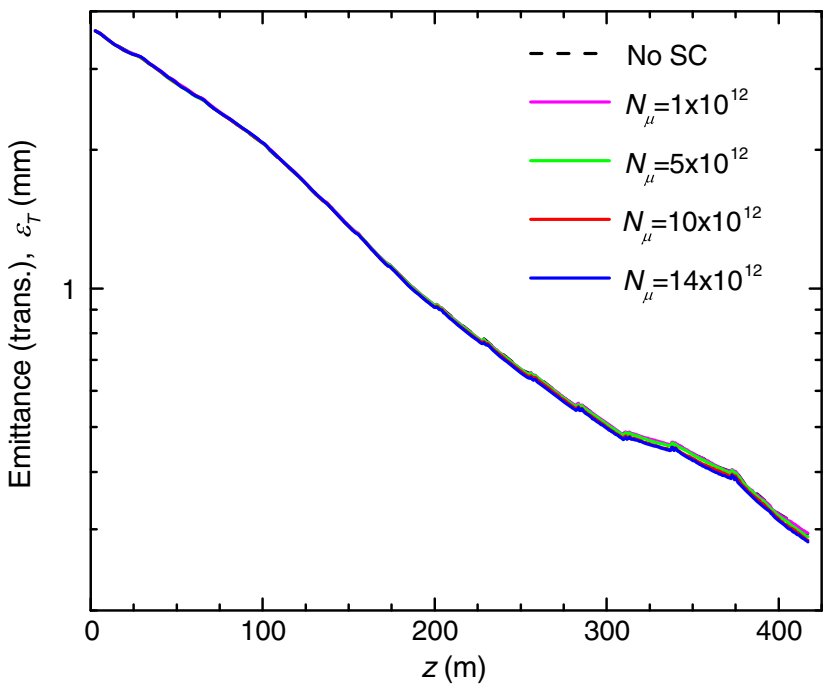

FIG. 4. Simulation results of the performance of the 6D cooling channel for a muon collider. The plot shows the evolution of the normalized transverse rms emittance as a function of distance along the channel for different beam intensities. At the end of the channel $(z=417 \mathrm{~m})$, with the assumption that space charge is negligible (dashed curve) the final transverse emittance is $\varepsilon_{T}=0.29 \mathrm{~mm}$. All $\mathrm{rf}$ cavities operate at $15.5 \mathrm{MV} / \mathrm{m}$.

axisymmetric ( $\mathrm{r}-\mathrm{z}$ ) simulation approach is much faster and simpler than a complete 3D simulation, which requires tilting the solenoids to generate necessary dipole fields and using complex-geometry absorbers for emittance exchange. The validity of this approximation was confirmed by later simulations with Warp [30], which showed that a complete 3D simulation resulted in no perceptible difference in the final result.

Before examining space-charge effects, a study was carried out comparing ICOOL and Warp results without space charge, checking correctness of the models. Good agreement between the two codes was found on the resultant emittance and transmission with and without muon decay. In particular, we found that the difference on the above parameters is within a 3\% error. A detailed study can be found elsewhere [24].

The input beam in the simulations, which contains 100000 particles, has a normalized transverse emittance of $3.62 \mathrm{~mm}$ and a normalized longitudinal emittance of $8.80 \mathrm{~mm}$, while the average longitudinal momentum is $209 \mathrm{MeV} / c$. Those parameters closely resemble the distribution of a muon beam at the entrance of the $6 \mathrm{D}$ cooling sequence after the merge [see Fig. 1(a)].

The evolutions of the transverse and longitudinal emittances as a function of distance by using the parameters from Table I are shown in Figs. 4 and 5, respectively. Without space charge (dashed curve), and after a distance of $417 \mathrm{~m}$ the 6D emittance has fallen by a factor of 1000 with a transmission of $50 \%$, including muon decay. In addition, at the end of the channel the transverse emittance

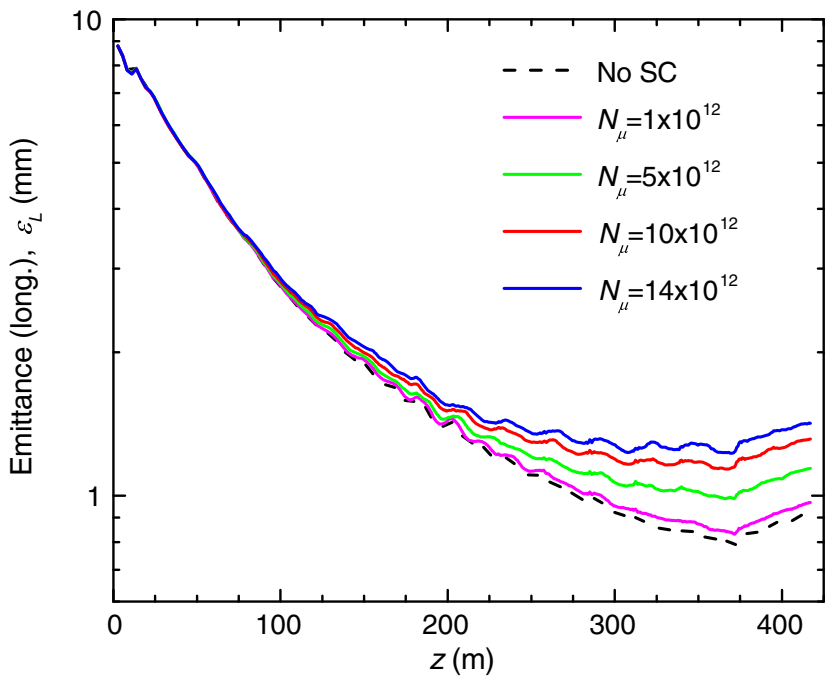

FIG. 5. Simulation results of the performance of the 6D cooling channel for a muon collider. The plot shows the evolution of the normalized longitudinal rms emittance as a function of distance along the channel for different beam intensities. At the end of the channel $(z=417 \mathrm{~m})$, with the assumption that space charge is negligible (dashed curve) the final longitudinal emittance is $\varepsilon_{L}=0.92 \mathrm{~mm}$. All $\mathrm{rf}$ cavities operate at $15.5 \mathrm{MV} / \mathrm{m}$.

decreased by a factor of $\sim 12$, while the longitudinal emittance shrank by a factor of $\sim 10$. The major beam parameters, at the entrance and exit of the channel, are summarized in Table II. Note that the normalized transverse and longitudinal emittances at the exit of the channel are 0.29 and $0.92 \mathrm{~mm}$, respectively. Thus, if the effect of space charge would be negligible, the muon collider emittance goal [7] at exit of the 6D cooling channel would be fully satisfied.

Next we attempt to estimate the number of muons in the bunch. Based on the published parameters of Ref. [31], a muon collider with a luminosity of $1 \times 10^{34} \mathrm{~cm}^{-2} \mathrm{~s}^{-1}$ at $1.5 \mathrm{TeV}$, will require bunches with $2 \times 10^{12}$ muons in the collider, or $\geq 5 \times 10^{12}$ muons at the end $(z=417 \mathrm{~m})$ of the

TABLE II. Beam properties at the entrance $(z=0 \mathrm{~m})$ and at the end of the post-merge channel $(z=417 \mathrm{~m})$. The values shown at the channel exit correspond to the optimum parameters for a muon collider and are the result of a simulation that assumes that the contribution from space charge is negligible.

\begin{tabular}{|c|c|c|}
\hline & $\begin{array}{l}\text { Channel } \\
\text { entrance }\end{array}$ & $\begin{array}{c}\text { Channel } \\
\text { exit }\end{array}$ \\
\hline Beam radius, $\sigma_{t}(\mathrm{~mm})$ & 27.1 & 2.1 \\
\hline Bunch length, $\sigma_{z}(\mathrm{~mm})$ & 80.0 & 18.1 \\
\hline Momentum, $P_{z}(\mathrm{MeV} / c)$ & 209.0 & 204.7 \\
\hline Normalized emittance (transverse), $\varepsilon_{t}(\mathrm{~mm})$ & 3.62 & 0.29 \\
\hline Normalized emittance (longitudinal), $\varepsilon_{l}(\mathrm{~mm})$ & 8.80 & 0.92 \\
\hline Minimum number of muons, $N_{\mu}\left(\times 10^{12}\right)$ & 10.0 & 5.0 \\
\hline Space-charge tune shift, $\Delta \nu_{s c} / \nu$ & 0.01 & 0.11 \\
\hline
\end{tabular}




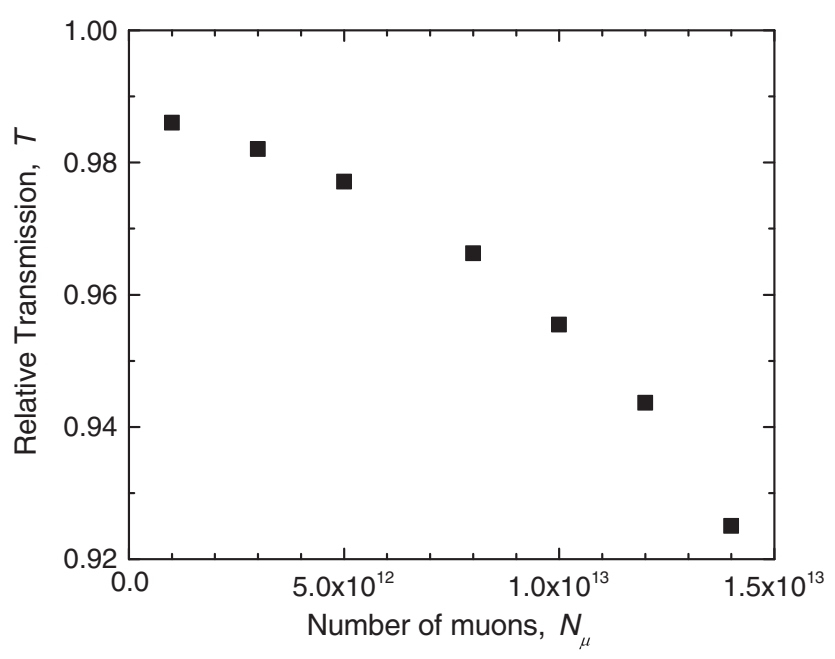

FIG. 6. Relative transmission, $T$ at the end of the channel $(z=417 \mathrm{~m})$ as a function of the beam intensity. Note that $T=1$ corresponds to the case without space charge. Parameter $N_{\mu}$ is the number of initial muons at the entrance of the post-merge channel $(z=0 \mathrm{~m})$.

post-merge $6 \mathrm{D}$ cooling channel. If we assume a $50 \%$ transmission as confirmed by our aforementioned simulations, we estimate the number of muons per bunch, $N_{\mu}$ at entrance of the channel $(z=0 \mathrm{~m})$ to be $\geq 10 \times 10^{12}$. Clearly this value indicates that space charge cannot be neglected and the implication of this will be discussed in more detail in the next paragraph.

Figures 4 and 5 also show the cooling efficiency with degree of beam intensity and the corresponding transmission for each case is shown in Fig. 6. Note that a transmission of 1 corresponds to the case without space charge. At the early stages $(z<100 \mathrm{~m})$ we see little difference in the emittances. However, at later stages, especially after $\sigma_{z}<40 \mathrm{~mm}$, cooling becomes strongly correlated to the number of muons in the bunch. This indicates that $N_{\mu}$ is the key parameter that governs the final emittance in an ionization cooling channel. Our simulations also predict that space charge is accompanied with a substantial particle loss which becomes more noticeable within the intensity regime of a muon collider. A salient feature of our study is that the transverse emittance is not affected by the bunch charge, suggesting that the deterioration of the cooling performance is primarily caused by the longitudinal space-charge force. This is not unexpected, since the force from the multi-Tesla magnetic field appears sufficient enough to balance the beam transversely. On the other hand, the space charge increases the length of the bunch, pushing more particles out of the $\mathrm{rf}$ bucket eventually leading to emittance growth and particle loss. Quantitatively, for a muon collider, longitudinal cooling below $1.3 \mathrm{~mm}$ is not viable under the current circumstances.

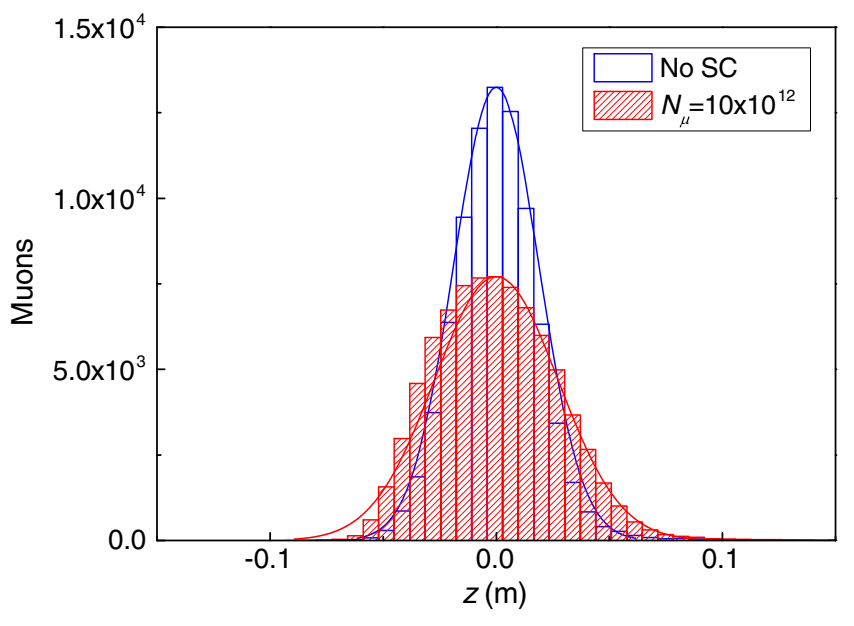

FIG. 7. Distribution of particles in the longitudinal direction at the end of the cooling channel: With the assumption that space charge is negligible $\left(N_{\mu}=1\right)$ and with $N_{\mu}=10 \times 10^{12}$ (red). The solid curves are fits into a Gaussian distribution.

Figure 7 displays the particle distribution in the longitudinal direction, at the end of the channel $(z=417.0 \mathrm{~m})$. If the effect of space charge is neglected (blue curve), the beam distribution is Gaussian with $\sigma_{z}=18.1 \mathrm{~mm}$. The influence of space charge is shown by the red curve. One can see that it defocuses the beam longitudinally, resulting to a bunch length increase to $\sigma_{z}=27.0 \mathrm{~mm}$. While in first approximation the distribution can be assumed to be Gaussian, it exhibits a slight asymmetry not observed by the red curve.

As noted earlier, space charge increases the length of the pulse, pushing more particles out of the rf bucket. One possible method to reduce this loss is to increase the rf gradient, which increases the depth of the bucket. In Fig. 8 we progressively raise the gradient from the initial design value of $15.5 \mathrm{MV} / \mathrm{m}$ and show results for two cases: one with $N_{\mu}=8 \times 10^{12}$ [Fig. $8($ a) $]$ and one with $N_{\mu}=12 \times 10^{12}$ [Fig. 8(b)] initial muons. As the rf gradient grows, the final achieved longitudinal emittance decreases and approaches the optimum cooling emittance of Table II, which is shown by the red half-filled circle. In addition, we find that the transmission improves by $\sim 5 \%$, for both cases, which fully compensates the loss from space-charge effect. Conversely, the transverse emittance is unaffected and remains mostly flat and close to the optimum value which is depicted by the red half-filled square. Our simulation also captures well the dependence of the compensation $\mathrm{rf}$ gradient with bunch charge. In particular, Fig. 8 shows that if the bunch intensity is increased by $50 \%$, the required gradient to push back to the optimum emittances is $20 \%$ higher. As a result, the space-charge force can be controlled by the rf gradient and the value for full compensation is correlated to the beam intensity. Since the nominal gradient for a normal conducting $805 \mathrm{MHz}$ rf cavity is $34 \mathrm{MV} / \mathrm{m}$ 


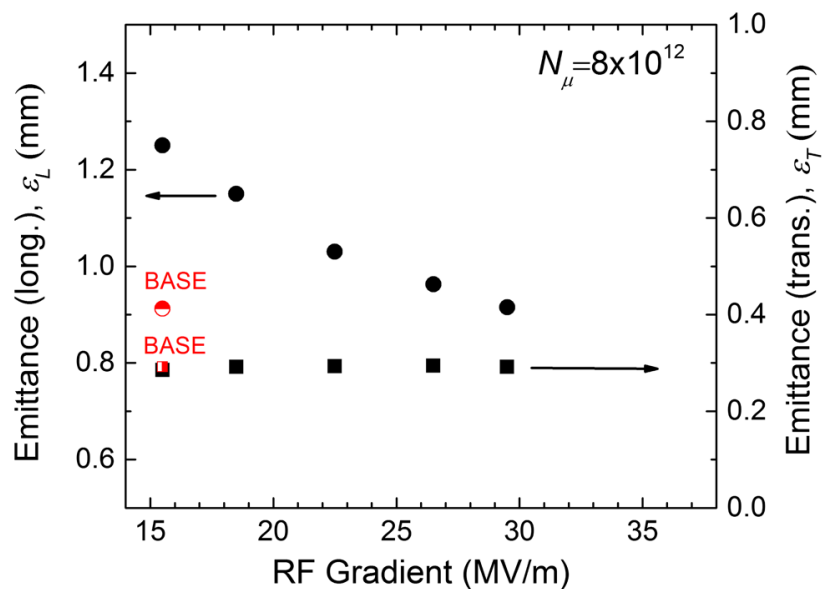

(a)

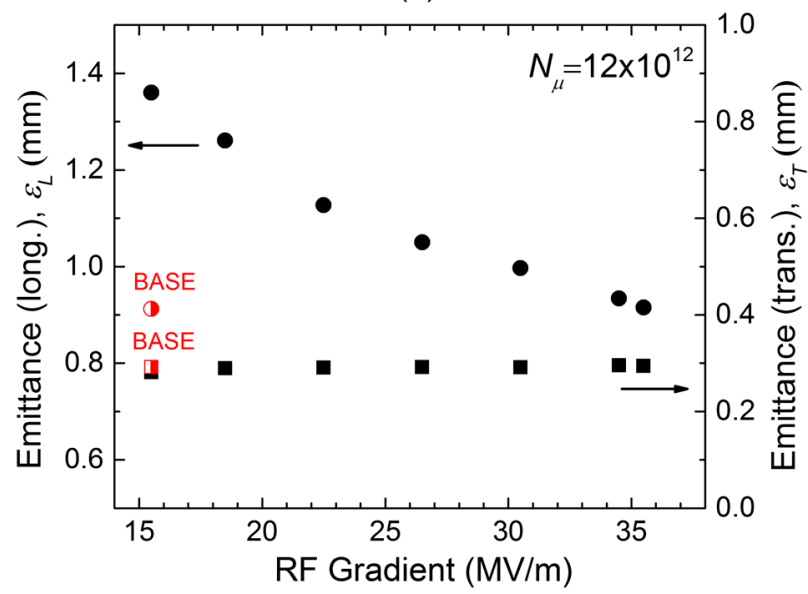

(b)

FIG. 8. Emittance values at the end of the channel $(z=417 \mathrm{~m})$ as a function of the $\mathrm{rf}$ gradient for (a) $N_{\mu}=8 \times 10^{12}$ initial muons and (b) $N_{\mu}=12 \times 10^{12}$ initial muons. The optimum transverse and longitudinal emittance values, the ones obtained by neglecting space-charge effects, are depicted by the half-filled squares and circles, respectively. We assume that all rf cavities along the channel operate at the same gradient.

[32], or less in the presence of a magnetic field [26,33], this fact implies numerous lattice design challenges.

To go beyond and get a quantitative prediction over a wide range of beam intensities, we plot in Fig. 9 (squares) the simulated rf gradient for space-charge compensation as a function of the bunch intensity. Next, to support our numerical findings we compare our results to the theoretical expression given by Palmer et al. [18]. Accordingly, if we ignore wall effects the longitudinal space-charge field can be expressed as follows [17]:

$$
E_{S C}=-\frac{e g_{0}}{4 \pi \varepsilon_{0} \gamma^{2}} \frac{\partial \lambda}{\partial z}
$$

where $\gamma$ is the relativistic factor, $\varepsilon_{0}$ is the dielectric permittivity in vacuum, $g_{0}$ is the geometric factor and $\lambda$ is the particle's line density. From the results in Fig. 7, if we

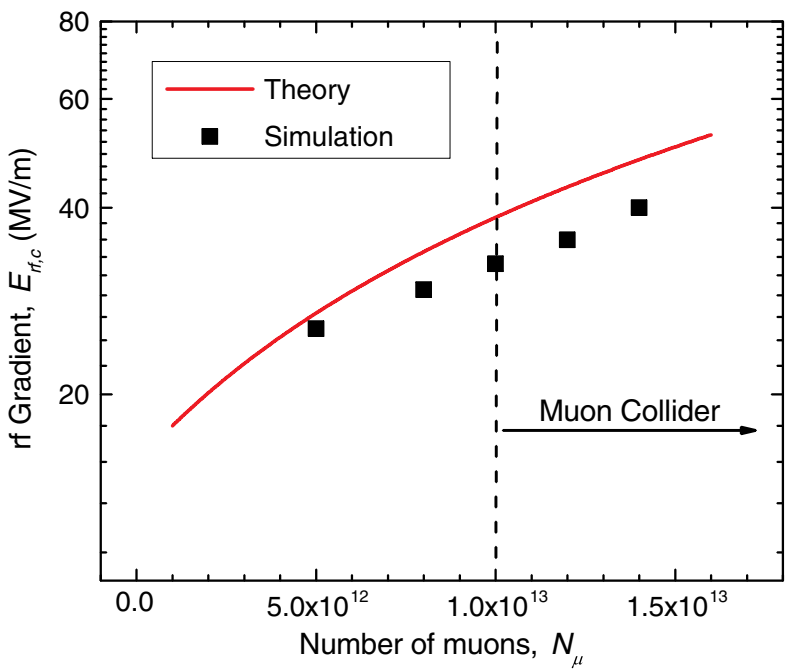

FIG. 9. Required gradient (solid curve) for a $805 \mathrm{MHz}$ rf cavity to reach the optimum value of $\varepsilon_{L}=0.92 \mathrm{~mm}$ at the end of the channel as a function of the bunch charge given by the expression (5). As before $N_{\mu}$ is the number of muons at the entrance of the post-merge $6 \mathrm{D}$ channel. The results of the Warp simulations (squares) show that for a muon collider in order to compensate space charge, the rf gradient needs to surpass $32.5 \mathrm{MV} / \mathrm{m}$, which is 2 times higher than the gradient assumed so far.

assume in first approximation the distribution to be Gaussian, then

$$
\lambda=\frac{N_{0}}{\sqrt{2 \pi} \sigma_{z}} e^{-\frac{z^{2}}{2 \sigma_{z}^{2}}}
$$

Here $\sigma_{z}$ is the bunch length and $N_{0}$ is the number of muons at the end of the channel. The beam expands in $z$ with a rate that is determined by the rate of change of the longitudinal space-charge field and can be expressed as follows:

$$
E_{S C}^{\prime}=\frac{d E_{S C}}{d z}=\frac{e N_{0} g_{0}}{4 \pi \varepsilon_{0} \gamma^{2} \sigma_{z}^{3} \sqrt{2 \pi}} .
$$

Note that Eq. (5) is evaluated at $z=0$ since for a Gaussian beam the defocusing from space charge is greatest in the core. In order to oppose the growth of the bunch, we need a compensation rf gradient, $E_{\mathrm{rf}, c}$ that should satisfy the following condition:

$$
\frac{d}{d z}\left[E_{\mathrm{rf}, c} \sin \left(\frac{2 \pi f}{c} z\right)\right]=E_{S C}^{\prime} .
$$

Here, $f$ is the rf frequency. From Eq. (6) we can estimate the compensation gradient to be

$$
E_{\mathrm{rf}, c}=\frac{e N_{0} g_{0} c}{4 \pi \varepsilon_{0} \sqrt{2 \pi} \gamma^{2} \sigma_{z}^{3}(2 \pi f n \cos \varphi)},
$$


where $\varphi$ is the rf phase and $n$ is fraction of the lattice filled with rf cavities. The geometric factor is determined by the beam radius $a$, and the pipe radius $b$ according to the relation [34] $g_{0}=1+2 \ln (b / a)$, with $b / a=3$ for our case. For the last cooling stage (stage 17) $f=805 \mathrm{MHz}$, and for the optimum beam parameters at the end of the channel from Table II, Eq. (7) predicts an rf gradient that is shown by the solid curve in Fig. 9. Clearly, for the intensity regime of a muon collider, the discrepancy between simulation and theory is near $10 \%$ with the latter predicting a higher gradient. While the wall effect has to be included to obtain a quantitative comparison, the theory provides a good first-order estimate of the required gradient. Equation (7) predicts that $E_{\mathrm{rf}, c} \propto 1 / \sigma_{z}^{3}$ suggesting that the bunch size is the crucial factor in controlling space charge. Based on Fig. 5, one potential solution would be to avoid longitudinal cooling to emittances $\leq 1.3 \mathrm{~mm}$. Another option is to compensate space charge by means of increasing the rf gradient. However, our numerical findings in Fig. 9 imply that for a muon collider in order to reach a longitudinal emittance that is $<1.0 \mathrm{~mm}$, the gradient of a $805 \mathrm{MHz}$ cavity needs to surpass $32.5 \mathrm{MV} / \mathrm{m}$.

\section{SUMMARY}

Although there has been a rapid progress in the design and simulation of 6D cooling channels for muon accelerators in the past decade, the influence of space charge in the whole design has been largely unexplored. This may become crucial towards the end of the cooling channel, where the muon bunch becomes short enough so that the interaction between individual particles can become important. In this paper, we have examined the influence of space-charge fields on the cooling process of muon beams. We showed that the cooling efficiency decreases with the degree of intensity, yielding to emittance growth and particle loss for beams with large intensities. A salient feature of our study was that the transverse emittance was not affected by the bunch charge, suggesting that the deterioration of the cooling performance is primarily caused by the longitudinal space-charge force. This is not unexpected, since the space charge increases the length of the bunch, pushing more particles out of the rf bucket eventually leading to emittance growth. Furthermore, we showed that the rf gradient can play a key role for spacecharge compensation in ionization cooling channels. With the aid of numerical simulations we established a quantitative relationship between the required compensation gradient and bunch intensity. We found that for a muon collider in order to obtain longitudinal emittances $<1.0 \mathrm{~mm}$, the rf gradient of a $805 \mathrm{MHz}$ cavity needs to surpass the demanding value of $32.5 \mathrm{MV} / \mathrm{m}$. Therefore, a potential solution would be to avoid longitudinal cooling to emittances $\leq 1.3 \mathrm{~mm}$.

\section{ACKNOWLEDGMENTS}

The authors are grateful to J.S. Berg, A. Friedman, J. C. Gallardo, I. Haber, M. Palmer, and R. Ryne for their support and for many useful discussions. This work is supported by the U.S. Department of Energy, Contract No. DE-AC02-98CH10886.

[1] D. Neuffer, Nucl. Instrum. Methods Phys. Res., Sect. A 350, 27 (1994).

[2] C. M. Ankenbrandt et al., Phys. Rev. ST Accel. Beams 2, 081001 (1999).

[3] M. M. Alsharo'a et al., Phys. Rev. ST Accel. Beams 6, 081001 (2003).

[4] G. I. Budker, in Proceedings of the 7th International Conference on High-Energy Accelerators, Yerevan, 1969 (Academy of Sciences of Armenia, Yerevan, 1970), p. 33.

[5] Yu. M. Ado and V. I. Balbekov, Sov. At. Energy 31, 731 (1971).

[6] A. N. Skrinsky and V. V. Parkhomchuk, Sov. J. Part. Nucl. 12, 223 (1981).

[7] R. B. Palmer, in Proceedings of the 23rd Particle Accelerator Conference, Vancouver, Canada, 2009 (IEEE, Piscataway, NJ, 2009), p. 652.

[8] R. B Palmer and R. C. Fernow, Six-dimensional bunch merging for muon collider cooling, in Proceedings of the 3rd International Particle Accelerator Conference, New Orleans, LA, 2012 (IEEE, Piscataway, NJ, 2012), TUPPR011, p. 1831.

[9] H. K. Sayed, R. B. Palmer, J. S. Berg, and D. Stratakis, Design and simulation of a high-field-low energy muon ionization cooling channel, in Proceedings of the 5th International Particle Accelerator Conference, Dresden, Germany, 2014 (JACoW, Geneva, Switzerland, 2014), p. 1386 .

[10] R. B. Palmer, "Muon Collider Parameters" Muon Accelerator Program Workshop (Menlo Park, CA, 2012) https:// indico.fnal.gov/conferenceDisplay.py?confId=5097.

[11] D. Neuffer, Part. Accel. 14, 75 (1983).

[12] A. Klier and G. G. Hanson, Neutrino Factory/ Muon Collider Document No. 298 (2004), http://nfmcc-docdb .fnal.gov/cgi-bin/DocumentDatabase/.

[13] J. Pasternak, Nucl. Phys. B, Proc. Suppl. 149, 271 (2005).

[14] Y. Derbenev and R. P. Johnson, Phys. Rev. ST Accel. Beams 8, 041002 (2005).

[15] P. Snopok, G. Hanson, and A. Klier, Int. J. Mod. Phys. A 24, 987 (2009).

[16] R. B. Palmer, Muon Accelerator Program Workshop (Menlo Park, CA, 2012) https://indico.fnal.gov/ conferenceDisplay.py?confId=5097.

[17] S. Y. Lee, Accelerator Physics (World Scientific, Singapore, 1999) .

[18] R. B. Palmer, Muon Accelerator Program Seminars (Upton, NY, 2011) https://indico.fnal.gov/conferenceDisplay .py?confId=4381.

[19] M. Aiba, M. Chanel, U. Dorda et al., Spacecharge compensation options for the LHC injector 
complex, in Proceedings of the 2007 Particle Accelerator Conference, Albuquerque, NM (IEEE, New York, 2007), p. 3390.

[20] J.-L Vay, D. P. Grote, R. H. Colen, and A. Friedman, Comput. Sci. Discovery 5, 014019 (2012).

[21] A. W. Chao and M. Tigner, Handbook of Accelerator Physics and Engineering (World Scientific, Singapore, 1999).

[22] R. B. Palmer, Neutrino Factory/ Muon Collider Document No. 250 (2002), http://nfmcc-docdb.fnal.gov/cgi-bin/ DocumentDatabase/.

[23] D. Stratakis, R. C. Fernow, J. S. Berg, and R. B. Palmer, Phys. Rev. ST Accel. Beams 16, 091001 (2013).

[24] D. Stratakis, R. B. Palmer, and D. P. Grote, Space-charge studies for ionization cooling lattices, in Proceedings of the 4th International Particle Accelerator Conference, IPAC2013, Shanghai, China, 2013 (JACoW, Shanghai, China, 2013), TUPFI088, p. 1553.

[25] V. Balbekov, Muon Accelerator Program Document No. 4365 (2013), http://map-docdb.fnal.gov.
[26] A. Moretti, Z. Qian, J. Norem, Y. Torun, D. Li, and M. Zisman, Phys. Rev. ST Accel. Beams 8, 072001 (2005).

[27] R. C. Fernow, in Proceedings of the 21st Particle Accelerator Conference, Knoxville, TN, 2005 (IEEE, Piscataway, NJ, 2005), p. 2651.

[28] map.fnal.gov.

[29] R. A. Kishek, Phys. Rev. Lett. 108, 035003 (2012).

[30] D. P. Grote, D. Stratakis, R. B. Palmer, and J. S. Berg, Space-charge limitations on the final stages of a muon collider cooling channel, in Proceedings of the 25th Particle Accelerator Conference, PAC-2013, Pasadena, CA, 2013 (IEEE, New York, 2013), THPHO13, p. 1331.

[31] R. B. Palmer, Draft muon collider parameters, Muon Accelerator Program Document No. 4318 (2011), http:// map-docdb.fnal.gov.

[32] D. Li, J. Corlett, A. Ladran et al., J. Phys. G 29, 1683 (2003).

[33] D. Stratakis, J. C. Gallardo, and R. B. Palmer, Nucl. Instrum. Methods Phys. Res., Sect. A 620, 147 (2010).

[34] S. Hansen, H. G. Hereward, A. Hofman, K. Huebner, and S. Myers, IEEE Trans. Nucl. Sci. 22, 1381 (1975). 